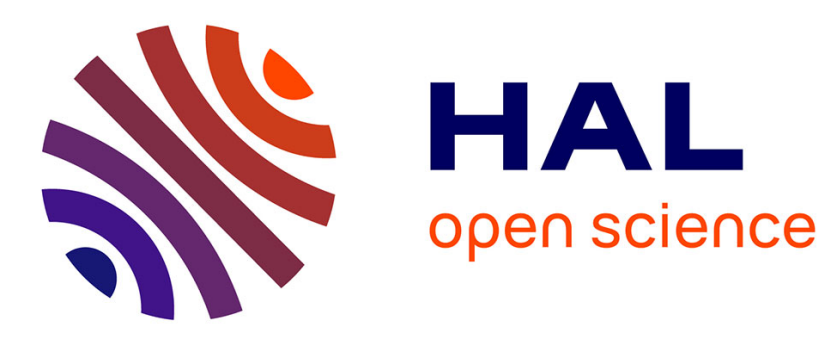

\title{
Le mouvement associatif islamique en Ile-de-France
}

\author{
A. Moustapha Diop, Riva Kastoryano
}

\section{To cite this version:}

A. Moustapha Diop, Riva Kastoryano. Le mouvement associatif islamique en Ile-de-France. Revue Europeenne des Migrations Internationales, 1991, 7 (3), pp.91-117. hal-01370810

\section{HAL Id: hal-01370810 \\ https://hal-sciencespo.archives-ouvertes.fr/hal-01370810}

Submitted on 23 Sep 2016

HAL is a multi-disciplinary open access archive for the deposit and dissemination of scientific research documents, whether they are published or not. The documents may come from teaching and research institutions in France or abroad, or from public or private research centers.
L'archive ouverte pluridisciplinaire HAL, est destinée au dépôt et à la diffusion de documents scientifiques de niveau recherche, publiés ou non, émanant des établissements d'enseignement et de recherche français ou étrangers, des laboratoires publics ou privés. 
Revue Européenne

des Migrations Internationales

Volume 7 - No 3

1991

\title{
Le mouvement associatif islamique en Ile-de-France*
}

\author{
A. Moustapha DIOP et Riva KASTORYANO
}

La dimension socio-religieuse a été la grande absente dans les recherches conduites depuis des décennies sur l'immigration non européenne ; des études dans ce domaine ont mis l'accent notamment, sur les conditions de logement, de travail, sur la scolarité des jeunes issus de l'immigration, sur les difficultés d'insertion de ces populations dans le moule français.

Vers la fin des années soixante-dix, la donne religieuse s'introduit timidement dans le champ de la sociologie de l'immigration (C. Hames, 1979). La " greffe " ne prend véritablement que lorsque les bruits et les fureurs parvenant de l'Orient proche ou médian rappellent la présence de populations musulmanes, déjà installées, et bricolant, vaille que vaille, avec le sacré.

Des questionnements vont s'agencer autour de l'appartenance religieuse et communautaire, autour des pratiques culturelles, des lieux de culte - « mosquées " -, et de leur fonction ; certaines études interrogent les prêches, les discours, afin de "déterminer les catégories de pensées", structurant l'intervention des musulmans dans le champ du politique (G. Kepel, 1985), et dans l'entreprise (C. de Wenden, J. Barou, T. Subhi, A.M. Diop, 1986).

Les recherches portant sur le mouvement associatif islamique sont peu nombreuses et restent parcellaires dans leur objet. Il est des études ayant trait aux modes sociaux de prédication (A.M. Diop, 1985) ; d'autres études décodent les formes d'action collective ( $R$. Kastoryano, 1988) ou analysent les stratégies de ces associations, en situant leur influence sur l'échiquier politique, local, national, voire international (G. Kepel, 1987, R. Kastoryano, 1988). Des esquisses sont dessinées dans certains articles, quant au rôle éducatif des associations (A.M. Diop, 1989), et ouvrages (G. Kepel, 1987, B. Etienne, 1987) ; elles donnent des indications, voire des pistes de recherche sur l'enseignement religieux, sur les maîtres, et sur les organisations islamiques internationales. 
C'est autour de la "fabrique " - l'expression est de G. Balandier - d'une identité musulmane, que des acteurs sociaux, engagés dans le mouvement associatif de type religieux tentent, d'une part de regrouper des musulmans, en diffusant et en valorisant la civilisation islamique, et d'autre part, de négocier avec les pouvoirs publics. En s'attribuant le rôle de gestionnaire de l'éducation des jeunes issus de l'immigration, les associations islamiques élaborent des politiques pour concurrencer, voire supplanter les associations de type laïque. En construisant des frontières de l'identité, les associations islamiques induisent-elles les populations en question vers un processus de marginalisation, ou favorisent-elles un schème d'intégration sociale?

\section{MÉTHODOLOGIE}

En 1986, G. Kepel (op. cit.) dénombrait 143 associations islamiques en lle-deFrance. En 1990, d'après nos recherches (fichier du CIEMI, J.O.), le nombre d'associations de type religieux est de l'ordre de 200. C'est pourquoi nous avions retenu un échantillon de 100 associations à enquêter ; le terrain a vite révélé que certaines de ces associations, soit n'existaient plus, soit avaient changé d'adresse - du reste non signalée au J.O. L'échantillon, pour des raisons de conjoncture, fut révisé à la baisse, et nous avons ajouté des associations non déclarées - au nombre de $8-$, du fait de leur richesse en contenu. L'échantillon actuel comprend 74 associations, dont 8 non déclarées ; toutefois le nombre des entretiens est de 82, puisque nous avons interviewé deux fois 8 associations - déclarées ou non -, afin de tester une hypothèse, suite aux événements dans le Golfe et la parution du livre de G. Perrault "Notre ami, le roi ". Nous avons revisité 4 associations informelles et 4 associations déclarées.

L'entretien était du type semi-directif, avec cependant des questions de type fermé - cotisations, nombre d'adhérents, fiche signalétique des dirigeants...( ('). Cinq langues ont été utilisées : le français, l'arabe, le turc, le peul et le cambodgien ; il est à noter cependant que plus de la moitié des entretiens s'est déroulée en français - selon le souhait des interviewés. Les entretiens ont duré en moyenne deux heures. Selon les cas, ils se sont déroulés en présence du bureau de l'association au grand complet; de deux membres du bureau; d'un dirigeant, assisté de l'imam et/ou du chargé des affaires religieuses au consulat (ceci est uniquement valable pour quelques associations turques); d'un dirigeant (cas le plus fréquent). Il est à noter que plus les questions étaient directes, plus les réponses étaient floues; l'obstacle a été toutefois franchi - en général -, soit par le biais de questions moins chargées, plus anodines dans. la formulation, soit par le biais de recoupements.

Cette étude, après avoir retracé la genèse et les motifs de création des associations, s'interroge sur leur mode de financement, décrit leur vie interne qui permet d'éclairer le rôle d'un acteur social décisif dans la vie associative de type religieux. Sites de mobilisation des ressources identitaires, les associations, au travers des discours de ses dirigeants, engagent différents types de tactiques et de stratégies afin de se situer tant sur le plan local que national de l'échiquier socio-politique. 


\section{GENĖSE ET MOTIFS DE CRÉATION DES ASSOCIATIONS}

Le mouvement associatif islamique constitue une véritable bouteille à l'encre ; les estimations, au début dans les années quatre vingt-dix, varient de 1000 à 1100 sur le territoire national. La loi du 9 octobre 1981 a joué sur le phénomène de création, conjuguée, il est vrai, à d'autres facteurs - manne pétrolière par exemple - (cf. G. Kepel, 1987).

Certaines associations ont pourtant pris racine dès le début du vingtième siècle ; c'est le cas de la Fraternité Musulmane, fondée en 1907 - deux ans après la loi de 1905 et six ans après celle de 1901, à Paris, et dont les objectifs s'agençaient autour de la solidarité et de la fraternité entre musulmans, de la protection des jeunes étudiants, nouvellement arrivés, du secours et de l'aide aux étudiants malades et nécessiteux, des services funèbres en cas de décès (Revue du Monde Musulman, Janvier-Mai, 1908). Dans les années trente, la Fraternité Musulmane - composée essentiellement d'intellectuels égyptiens - a participé activement aux préparatifs et aux travaux du Congrès des Musulmans en Europe, tenu à Genève en 1935 (M. Kramer, 1986). Dans les années cinquante, l'Union Générale des Etudiants Musulmans Algériens a pris la relève, et dans les années soixante, ce fut le tour de l'Association des Étudiants Islamiques, devenue après l'Association des Étudiants Islamiques en France (AEIF) créée sous l'égide d'un Pakistanais, chercheur au CNRS, Muhammad Hamidullah (G. Kepel, op. cit.). C'est à partir des années 1960 que les travailleurs immigrés entrent timidement dans le champ associatif de type religieux.

D'après notre étude, sur un échantillon de 66 associations déclarées (cf. tableau 1 à 9), 53 d'entre elles l'ont été entre 1981 et 1990, 11 entre 1970 et 1980 , et 2 entre 1960 et 1969 . S'agissant des associations de fait, elles sont presque toutes nées entre 1980 et 1987, seule la Jawiha Cheikhna Hamilulah, association d'obédience confrérique a été fondée en 1975.

Les raisons invoquées dans la création des associations - déclarées ou non - sont indiquées dans le tableau ci-dessous :

TABLEAU 1 : Motifs de création

\begin{tabular}{|l|c|}
\hline \multicolumn{1}{|c|}{ Motifs de création } & Nombre d'associations \\
\hline $1-$ Reconnaissance juridique & 17 \\
\hline $2-$ «Sauver les enfants " & 13 \\
\hline $3-$ Le « Réveil des adultes " & 11 \\
\hline $\begin{array}{l}4-\text { Motivation purement religieuse } \\
\text { - rassemblement pour prières et réflexion (10) } \\
\text { - prolongement d'une confrérie mission (10) }\end{array}$ & 20 \\
\hline $5-$ Evènements & 5 \\
\hline
\end{tabular}




\begin{tabular}{|l|c|}
\hline $6-$ Scission & 3 \\
\hline $7-$ Assistance sociale & 3 \\
\hline $8-$ Autres & 2 \\
\hline TOTAL & $\mathbf{7 4}$ \\
\hline
\end{tabular}

Il est entendu qu'il existe des interrelations entre les différents thèmes évoqués ; une raison peut en recouper deux ou plusieurs autres, toutefois, les interviews font dans la plupart des cas ressortir le " facteur principal » de la création de l'association, même s'ils en rajoutent d'autres. Pour la clarté, nous avons retenu le " facteur principal ", décliné par chaque interviewé. En grossissant les traits, on se trouve entre deux pôles : un pôle de l'offre et un pôle de la demande d'islam ; entre ces deux extrémités oscillent des points.

L'offre peut s'expliquer par l'application de la loi 1901 qui donne une reconnaissance juridique à un rassemblement informel. Sur 17 associations, 13 d'entre elles spécifient qu'elles ont été créées : soit pour être reconnues par les autorités locales ; le cas-type est celui de l'association Intégrité ; soit pour pouvoir acheter ou louer un local ; soit encore pour sortir de l'informel ; ou bien c'est parce qu'il existe un canevas juridique simple. Pour le reste de l'échantillon, les motifs de création sont divers et variés : il est des créations pour des raisons d'ordre pratique ; pour d'autres, la nécessité de " représentativité " a été un facteur déclencheur.

D'autre part, l'offre vient des musulmans, et est destinée à d'autres musulmans ; elle est " multiforme " : ceci va du rassemblement spontané (prières) jusqu'à la " pression communautaire " qui amène les moins décidés à se définir et à passer à l'acte. Si la confrérie recrute en principe dans un milieu endogène fermé, les missionnaires, au contraire, s'adressent au plus grand nombre ; toutefois, certains, pour des raisons de conjoncture évoluent dans un champ restreint (Ahmadiyya France) ; d'autres missionnaires, en revanche, labourent allègrement le terrain en friche (Foi et Pratique).

La " demande d'islam » est générée et sollicitée par les adultes " qui ont assez de faire la fête " et/ou qui " se sont rendus compte qu'ils sont là pour un bon bout de temps et qu'ils ne sont plus de passage ". Ce constat dressé a eu une valeur heuristique sur les attitudes et comportements des adultes qui se sont " réveillés" pour eux-mêmes et pour " sauver les enfants".

Ce " réveil " des adultes procède de plusieurs causes : destructuration de la cellule familiale, la déception qui va susciter un repli, un retrait vers des valeurs traditionnelles, ou la déconvenue à l'endroit de l'école laïque, qui ne prend pas en compte la dimension religieuse. D'autres parents ont entendu l'« Appel de l'islam ", par le biais du mouvement Foi et Pratique.

"Sauver les enfants ". Le jeune issu de l'immigration sert de fonction miroir dans la topographie familiale ; spectateur plus qu'acteur, il assiste au bricolage, 
selon l'expression de C. Lévi-Strauss, mis en chantier par ses parents. Ceux-ci, privés de l'efficacité de la tradition silencieuse du pays d'origine, où toute la communauté participe à l'éducation des enfants, engagent de différents schèmes de socialisation fondés soit sur des éléments sélectifs de tradition et de modernité, soit sur l'éducation "pure et dure ", ou encore sur l'éducation de la société d'accueil. C'est, semble-t-il, parce que ces divers types de socialisation s'avèrent déficients, voire inopérants que les parents sinterrogent sur le devenir de leurs enfants après avoir procédé à une autocritique exprimée en termes de négligence qui porte pour certaines catégories sur l'inculcation de la langue, de la culture. Pour d'autres, la crise est plus profonde, plus totale, " on était en train d'en faire de petits Français ". D'autres encore mettent l'accent sur le clivage culturel entre parents et enfants. A la suite de ce constat, des parents sont passés à un début d'action " on s'est décidé à faire quelque chose pour nos enfants".

D'autres individualités - notamment dans les associations turques, portent la critique non sur la société d'accueil, mais plutôt sur les étrangers eux-mêmes, et proposent " pour sauver notre jeunesse ", d'éviter le " modèle arabe ". Si la confusion des identités est une réalité, elle doit cependant n'être que momentanée, d'où la nécessité de procéder au " bon choix " pour les jeunes.

Entre l'offre et la demande, d'autres motivations de création d'associations ont été avancées par les enquêtes : l'impact de certains événements (l'invasion soviétique, l'effet Le Pen, l'effet Rushdie, le "foulard islamique ", les querelles communautaires, la bataille pour la représentativité) ; les problèmes de scission (mésentente, ambitions personnelles, sous couvert de recherche d'un nouvel espace), l'aide sociale, (l'éducation sanitaire des femmes, l'accueil de jeunes filles, l'assistance ainsi que le soutien familial) et d'autres questions - mimétisme, réalisation d'un " testament".

\section{INITIATIVE DE LA CRÉATION ET DIFFICULTÉS RENCONTRÉES}

A la question " qui est à l'initiative de la création de l'association ? ", 27 associations déclarées ont répondu que la fondation procède d'une initiative personnelle, tandis que pour 37 associations, elle est le fait soit de groupes d'amis, de nationaux, ou de collègues de travail, soit de groupes d'associations (Fédération Nationale des Musulmans de France ; Union des Organisations Islamiques de France; Fédération des Associations Islamiques d'Afrique, des Comores et des Antilles. Seule une famille a pris la décision de créer d'elle-même une structure associative (Vivre l'Islam en Occident). Pour les associations de fait, il y a plus d'initiatives personnelles que de groupements de personnes.

Quant aux difficultés rencontrées au moment de la création, $3 / 4$ de l'échantillon des associations déclarées admettent n'en avoir rencontré aucune, que ce soit avant ou après la loi du 9 octobre 1981, tandis le quart de l'échantillon met l'accent sur les problèmes suscités lors de la création. Ces problèmes sont soit d'ordre matériel (location ou vente d'un local), soit sur l'image négative de l'islam, soit sur le manque d'expérience (barrière linguistique). Les problèmes inter-musulmans se scindent en : méfiance des parents vis-à-vis du cursus culturel proposé par les 
associations islamiques ; règlements de compte entre « harkis » et populations musulmanes.

\section{Ressources des associations}

Les associations indiquent que leur budget provient, selon les cas, de cotisations, dons et collectes, de l'aide des États, et des organisations internationales. A quelques variantes près, la technique d'incitation aux dons est identique d'une association à une autre - quelle que soit du reste sa taille; elle s'échelonne sur trois niveaux : l'association s'adresse à ses membres - c'est la phase interne, ensuite, elle s'ouvre vers l'extérieur immédiat, soit en prenant contact avec des associations de même sensibilité, soit en procédant au porte-à-porte (visite chez des individualités, chez des commerçants). L'association, dans la troisième phase d'exploration, investit des lieux plus lointains (Paris, et autres villes situées dans un rayon raisonnable). Les associations gérant une salle de culte, bénéficient, elles, d'autres atouts : c'est d'abord la caisse du " denier du culte " placée dans le lieu de prière, c'est ensuite, les jours de fêtes (les deux Ayds), ou le vendredi, périodes pendant lesquelles, les pratiquants contribuent, spontanément, sans aucune sollicitation externe, aux " impôts " hebdomadaires ou annuels. Les collectes destinées à l'achat d'une salle de prière ou d'un terrain pour la construction d'une mosquée, ou alors aux grands travaux, empruntent le même système que celui de l'incitation aux dons, en y ajoutant un périple dans les pays limitrophes, et/ou dans des pays musulmans, après constitution d'une commission ad hoc.

Le financement des associations islamiques par des États étrangers est sujet d'interrogations scientifiques, de gloses et de controverses (G. Kepel, op. cit. 211, 223 ; Le Nouvel Observateur : "Islam, les financiers de l'intégrisme ", 19-25 juillet 1990). Comment les associations se définissent-elles par rapport aux aides extérieures ?

21 associations déclarées sur 66 , c'est-à-dire près de $30 \%$ se sont exprimées de façon plus ou moins explicite sur la question ; on se trouve en présence de plusieurs cas de figure:

- Les associations qui acceptent pleinement le principe de l'aide extérieure ; c'est le groupe dominant (10 associations), qui justifie le soutien financier accordé ou pas, au nom de la solidarité islamique ; celles qui admettent le principe, sans y adhérer, et, ce afin de garder une liberté de parole ou celles, qui n'y adhèrent pas, pour des raisons d'ordre pragmatique et stratégique (FNMF);

-. Les associations (au nombre de 6) farouchement opposées à toute aide, comme par exemple, l'Association culturelle islamique d'Epinay : "Il y a beaucoup d'associations financées par les organisations, telles que la Ligue islamique, la Mosquée de Paris, ou par les États comme l'Arabie Saoudite ou l'Iran. Mais pour nous, c'est clair, dès le départ, aucun financement de n'importe quel État ". Mais elles admettent, cependant, d'être éventuellement subventionnées par la France.

Les associations ( 3 d'entre elles), restées plutôt sceptiques, quant aux aides, parce que déçues par de vaines démarches. 
TABLEAU 2 : Origine des aides et associations destinataires

\begin{tabular}{|l|c|c|c|c|c|}
\hline $\begin{array}{c}\text { Associations } \\
\text { Origine des aides }\end{array}$ & France & Maghrébines & $\begin{array}{c}\text { Negro- } \\
\text { africaines }\end{array}$ & Pluri-Nat & Autres \\
\hline $\begin{array}{l}\text { Pays et organisations } \\
\text { internationales }\end{array}$ & & & & & \\
\hline Pays du Golfe & 1 & 3 & 2 & 1 & - \\
\hline Grand Maghreb & & 1 & 1 & & \\
\hline Grande-Bretagne & & 1 & 1 & & \\
\hline $\begin{array}{l}\text { Organisations } \\
\text { internationales }\end{array}$ & & & & & 1 \\
\hline
\end{tabular}

Quant aux aides, elles sont de différents types ; elles peuvent être soit en nature (livres, cassettes), soit en allocations de bourses d'études, ou encore en espèces. 10 associations (plusieurs réponses) notifient la provenance de leurs aides : deux ont leur origine au Maghreb (Libye, Maroc) ; 7 viennent des pays du Golfe (Arabie Saoudite, Koweit) et une, de la Grande-Bretagne (voir tableau).

\section{FONCTION DES ASSOCIATIONS ET STRUCTURATION DES APPARTENANCES}

Les associations se donnent pour fonction d'investir tout autant le domaine socio-culturel que celui de l'éducation religieuse, de l'assistance sociale, ou celui de la défense des intérêts. La division des tâches ne semble pas aussi accusée, puisque le religieux est considéré comme un "phénomène social total ", et comme tel, il englobe tous les paliers de la vie, ainsi que l'atteste l'association Vivre l'Islam en Occident (VIO) : " on ne peut pas dire que la branche d'un arbre est sans rapport avec le tronc. Les cinq piliers de l'Islam, c'est le tronc vertical, après cela, le culturel, le social, ça va être les branches qui vont partir de ce tronc, mais qui ne peuvent partir d'autre part que de ce tronc. Si ça ne part pas de ce tronc, c'est un bois mort, et elles n'ont aucune fécondité, aucune justification possible. Donc c'est lié. Et toute construction peut être valable, mais elle n'est pas islamique ".

La " construction" suivante n'a que valeur de proposition : 
TABLEAU 3 : Typologie des fonctions (plusieurs réponses)

\begin{tabular}{|l|c|}
\hline \multicolumn{1}{|c|}{ Fonctions } & Nombre d'associations \\
\hline 1. Education & 94 \\
2. Assistance sociale et religieuse & 32 \\
3. Dévotion & 15 \\
4. Information et dialogue & 11 \\
5. Mission et prosélytisme & 9 \\
6. Coordination & 5 \\
7. Défense des intérêts & 5 \\
8. Réflexion & 4 \\
\hline
\end{tabular}

Les fonctions éducatives constituent la préoccupation principale des associations déclarées ou de fait ; le thème éducatif comporte deux volets, liés ou séparés selon les cas : d'une part, l'enseignement d'une ou dans une langue (arabe, français, turc, urdu...) et le soutien scolaire, et d'autre part, l'éducation religieuse.

Les associations de l'échantillon représentent un quasi microcosme de la " communauté musulmane " en France ; elles sont clivées en sunnites et chiites. Ceux-ci sont d'origine irakienne (groupe Ahl Al Beit), britanno-pakistanaise (Imania organisation), ou française de l'Inde et de l'Indochine (Association indo-française des Jaffari ; Association islamique des Français de l'Inde et de l'Indochine). Les sunnites sont maghrébins, français, turcs, négro-africains.

Au-delà de cette différenciation apparaissent d'autres tendances, au nombre de quatre :

- les associations, appendices d'ordre sufi (confréries),

- les associations "piétistes et communautaires",

-... les associations, affiliées aux missions religieuses des consulats,

- une " secte ", la Ahmadiyya, dont le fondateur, Mirza Ghulam, mort au début du siècle, se voulait être la synthèse du Mahdi des musulmans, du messie des chrétiens, et de l'avatar (incarnation) de Krishna.

Les associations-confréries, au nombre de six traversent les différentes nationalités : turque (association Amitié Solidarité de Mevlana), franco-algérienne (Les Amis de l'Islam, adeptes de la Alawiyya), malienne et sénégalaise (Jawiya cheikh Hamilulah; Voie Tijaniyya ; NDiaz-Layenne, et les murid du Mime et de l'association des serviteurs de l'Eternel).

Les associations de type piétiste sont représentées par Foi et Pratique ; quant à celles, affiliées au consulat, elles sont exclusivement turques. 


\section{LES DIRIGEANTS}

Dans l'ensemble, les dirigeants sont, pour 50 d'entre eux dans la catégorie " deuxième âge ", et 23 dirigeants sont soit en situation de pré-retraite, soit à la retraite. Un dirigeant n’a pas décliné son âge.

Si les $3 / 4$ sont ou ont été des ouvriers spécialisés, le quart des dirigeants, compte non tenu des étudiants, exercent ou ont exercé des métiers de secrétaire de direction, d'avocat, d'ancien fonctionnaire, de médecin, de chef d'entreprise, d'écrivain-journaliste ou d'éditeur.

Plus de la moitié des dirigeants ont un niveau d'éducation religieuse faible, voire très faible ; le niveau d'instruction ne recoupe pas forcément les tranches d'âge ; socialisés au pays d'origine ou en France, ils ont une connaissance sommaire, se limitant, dans le meilleur des cas, à quelques sourates du Coran. Ceux qui disposent d'un niveau moyen ont suivi des études coraniques ou théologiques au pays, ou ont " découvert et connu l'islam en France " et " appris véritablement l'arabe littéraire en France ". Parmi ces autodictates, se trouvent des Français convertis, des jeunes issus de l'immigration, des Marocains, des Négro-Africains. Le niveau d'études supérieures traverse quelques nationalités: sénégalaise, syrienne, marocaine, turque, malienne. La majorité a suivi d'abord un cursus dans des écoles coraniques ou des medersas ou des instituts théologiques dans le pays d'origine ; certains, chez les Négro-Africains, ont poursuivi des études soit au Maghreb, soit au Moyen-Orient, ou en Arabie Saoudite. Quelle que soit la nationalité, près du tiers de ces dirigeants sont issus de familles de lettrés musulmans chez les Négro-Africains.

\section{LES IMAMS}

Limam devient un personnage incontournable dans la vie associative, en tant que gestionnaire du spirituel. "Célébrer les prières quotidiennes, prononcer le prône du vendredi et donner des cours d'arabe, de religion ", telle est, selon l'Association de la communauté islamique (Yvelines), par exemple, la tâche d'un imam.

Sur un échantillon de 69 associations - les organisations féminines (au nombre de 5) n'ont pas d'imam -, 53 associations entretiennent officiellement des imams rémunérés ou bénévoles : 46 imams pour les associations déclarées, et 7 , pour les associations de fait. Les imams rémunérés d'associations déclarées relèvent de trois catégories : ceux qui sont envoyés et rémunérés par des États d'origine (dans notre échantillon, seules sont concernées 4 associations turques, mais en fait le Maroc et l'Algérie envoient aussi des imams à ses ressortissants); ceux qui sont recrutés et payés par un État tiers (une association maghrébine a un imam payé par la Libye, sans contrepartie); et ceux qui travaillent pour le compte d'associations, soit en occupant parallèlement des fonctions dans le bureau, soit en restant un simple membre ou un sympathisant de l'organisation. En ce qui concerne les imams bénévoles - en fait certains reçoivent des dons venant des fidèles ..., ils représentent, dans la plupart des cas la garantie morale des associations, et leurs meilleurs agents publicitaires pour drainer d'éventuels membres. 
Pour les associations de fait, la charge d'imam échoit selon les cas, au représentant du guide confrérique, soit à la personne la plus disponible, disposant d'un bagage religieux - c'est le cas pour les deux foyers en Seine-Saint-Denis. L'enquête révèle, d'autre part, la présence ou la circulation d'une autre catégorie d'imams, à savoir les " occasionnels", qui maîtrisent des sourates, dirigent les prières quotidiennes, et les "épisodiques ", envoyés, au moment des fêtes, tel le Ramadan, par la Mosquée de Paris à certaines associations de même obédience. Le choix ou la cooptation des imams par les associations s'analyse à travers quelques cas de figure : par affinité, par confiance, par une heureuse rencontre fortuite, pour combler un vide, ou pour responsabiliser les convertis. Des imams rémunérés se trouvent souvent dans une situation de précarité, soumis qu'ils sont soit aux aléas financiers de leurs employeurs, soit à la législation en vigueur en France.

La question des imams interpelle les associations doublement. D'une part, ceux-ci ont été, pour la plupart, socialisés dans leurs pays d'origine, méconnaissant les arcanes de la société française, et sont souvent assez éloignés sur le plan pédagogique des attentes des jeunes issus de l'immigration ; d'autre part, certains jeunes " réislamisés " se sont formés eux-mêmes pour devenir imams - le cas des Minguettes. Pour parer à ce dysfonctionnement, l'ACMIF propose une formation complète d'imams, originaires de France. Deux associations, l'une malienne (ATMRF), l'autre sénégalaise (ARAI) essaient, elles aussi, de leur côté d'apporter des éléments de réponse à cette "grande question"; si l'ATMRF investit les foyers de travailleurs pour former des imams, l'A RAI, quant à elle, met l'accent sur la formation des jeunes. (Diop 1989). A la quête de savoir s'il faut aller jusqu'au Caire ou en Syrie, l'association Foi et Pratique, elle, opte aussi pour " ici et maintenant ", avec son projet de création d'un institut théologique en banlieue parisienne.

\section{DISCOURS DES ASSOCIATIONS : ISLAM ET SOCIÉTÉ FRANÇAISE}

« Dans la rencontre des cultures, la religion est le dernier bastion qui refuse de se rendre " affirme R. Bastide. La mise en parallèle des différents types de discours sur l'islam permet de reconstruire la perception qu'ont les associations de leurs religions et de la société française. Au travers des discours se dessinent des modèles d'attitudes, voire des stratégies pour "défendre la bannière ", soit pour vivre en vase clos, ou d'une manière plus ouverte, ou alors pour proposer une rencontre entre deux visions du monde.

En exergue, des associations, comme par exemple UIF (association turque) introduisent pour baliser le terrain, une définition de l'homo islamicus : "le musulman veut dire celui qui est soumis à l'ordre de Dieu, qui respecte les autres, et qui est tolérant "; et comme tel, il peut vivre et s'adapter dans n'importe quel milieu, inspiré par le modèle du Prophète qui " vivait au début dans une tribu mécréante " (AUI) ; s'il est difficile d'affirmer sa foi, comparativement au pays d'origine, le musulman se doit de garder l'assurance. 
L'affirmation de sa foi passe par le truchement d'une vie intérieure intense, car "l'islam, c'est que que tu vis " (Les Amis de l'islam), mais aussi dans la mise en relation avec d'autres personnes c'est une religion ayant pour soutènement la mansuètude, surtout à l'égard de ceux qui " ne sont pas tolérants avec nous " (Jamaat Fath). Vivre l'islam en France est diversement apprécié par les associations ; pour les unes, il ne peut exister aucune contrainte à ce sujet, puisque " en France, on n'interdit pas à quelqu'un de pratiquer sa religion ; la loi protège au contraire la pratique religieuse " (Association islamique et culturelle des marabouts) ; pour d'autres, cette action procède de l'échange de bons procédés, puisqu' " au Maroc, il y avait toujours des églises pour eux, et personne ne les a jamais empêchés de pratiquer leur religion " (association informelle Bobigny).

Bien que deuxième religion en France, l'Islam n'est pas " reconnu ", et " la présence islamique n'a jamais été acceptée par la majorité des Français " (Association islamique Boulogne). Et pourtant, comparativement à d'autres communautés, les musulmans n'ont à aucun moment porté tort à ce pays. Mal aimé et rejeté, l'Islam sert, à l'occasion, de bouc-émissaire, en plus. L'amalgame entre terrorisme et religion musulmane est fallacieux, et signe de non-reconnaissance de l'autre : des associations restent persuadées que l'image négative relève de l'infra-droit : « il faut que l'islam soit reconnu comme une religion, comme le judaisme, le protestantisme, le catholicisme ; il n'a pas encore joui de ce droit comme d'autres religions " (UIF). Ceci est intimement lié au statut d'immigré non-européen " Nous, musulmans - pratiquants ou pas, nos noms, nos prénoms revendiquent que nous sommes musulmans " (UIF). Cette politique de " deux poids, deux mesures " se vérifie à différents paliers de la vie institutionnelle ; au niveau de l'application des lois, et au niveau des pratiques administratives.

Cette manière assez cavalière de traiter l'islam peut induire des réactions qui surgissent à l'occasion d'événements externes ou internes; pour une association de jeunes, le retour au religieux de ses collègues provient de la conjugaison de deux facteurs, d'abord " l'avènement de la République d'Iran ", qui a " redonné un élan ", parce que " avant, parler de l'islam, c'était presque honteux ; je crois que la République islamique a rendu une fierté, en quelque sorte que les gens avaient perdue "; ensuite, les jeunes sont allés à la quête des racines, parce qu'ils " ont compris qu'on les a roulés : le gouvernement et toutes les associations porteparole, auto-désignés, SOS Racisme, France Plus ". Pour d'autres groupes informels, par ex. - celui des femmes de la Mosquée de Paris - " l'affaire des foulards a fait venir beaucoup de musulmanes, car elles se sont senties agressées, et ont éprouvé le besoin de se réunir, de se regrouper ". Les affaires (Rushdie, foulard) ont montré, selon l'ACMIF, la maturité, et l'esprit de tolérance des musulmans. « Nous avons pris plein de gifles : Rushdie, foulards, refus pour implanter un lieu de culte... Alors ceux qui nous accusent d'intégristes, d'être peu tolérants, pourraient un peu modifier leur appréciation ". Et pourtant, ces " affaires " mais surtout celle du foulard, ont laissé dans bien des familles un souvenir plutôt amer :

"Dans une école maternelle, des jeunes ont été mises à la porte parce qu'elles portaient le foulard... Ici, je connais des mères de familles qui travaillaient depuis des années comme femmes de ménage dans des différents organismes publics, qui, du jour au lendemain, se sont trouvées à la porte, avec leurs gosses, sans res- 
sources. Simplement parce quil y a eu cette crispation. Ce genre de crispation, il faut réussir à la désamorcer, avant qu'elle puisse se concrétiser " (VIO).

A l'image d'un islam intolérant et semeur de trouble, des associations opposent un islam "facteur de stabilité et de paix sociale". Une telle proposition, emprunte d'abord la voie de la critique : " on dérange, et on (le gouvernement) préfère une intégration à la Harlem Désir ou à la Areski Dahmani, à celle conforme à l'islam, à la religion. Je ne suis pas sûr que Désir ou Dahmani donnent à ces jeunes des principes qui leur permettent de vivre correctement " (FNMF). Pour le porte-parole de cette fédération, la " révolution des lycéens " ne touche que les jeunes, n'ayant reçu aucune base d'éducation religieuse, qui contestent non pas le manque de matériel et de confort dans les lycées, mais plutôt leur mal-vie. Alors que " nos jeunes ne font pas partie de cette contestation, puisqu'on les éduque sur la base des lois islamiques, selon lesquelles " il faut se contenter de ce que Dieu te donne, et tu seras parmi les meilleurs hommes ".

En lieu et place d'une socialisation qui ne donne plus de résultats tangibles, les associations, elles, bien que démunies (enseignement dans des lieux insalubres), offrent aux jeunes une "philosophie qui leur permet de se réaliser ". Non seulement à l'école, mais aussi dans les îlots sensibles interviennent des associations "Prenez l'exemple d'un quartier qui a défrayé la chronique : Les Minguettes, eh bien, tous les jeunes du quartier ont rejoint la Jamaât (Foi et Pratique); depuis le quartier s'est calmé. C'est le triomphe de l'islam et de la paix. On joue un rôle social important. Les jeunes étaient abandonnés, nous sommes allés vers eux ; nous les avons récupérés grâce à nos actions. Nous avons osé aller voir ". Autant de liberté de mouvement et de parole, tout cela, semble-t-il, inaugure d'une cohabitation, certes houleuse, mais n'arrivant pas au drame puisqu'il y a « en France la possibilité d'avoir des gens intelligents, et qui peuvent aider à faire évoluer la situation " (Association des Droits de l'homme en Islam). En tout cas, dans la cité des papes, des petites filles ont manifesté, contre la " crispation ", leur solidarité à une camarade marocaine, renvoyée de son école, pour avoir porté le foulard : “ (...) et l'après-midi ses camarades françaises, pas du tout d'accord, sont venues avec un foulard à l'école. C'était un petit peu (...) une certaine vocation au respect à la différence, au respect des diversités (...) " (VIO).

La France, une chance pour l'islam ? ou l'islam, une chance pour la France? Le débat reste ouvert, tandis que des associations dénoncent le " narcissisme des petites différences " traversant la "communauté islamique "; certaines d'entre elles s'interrogent sur la dichotomie islam/musulman « les musulmans et l'islam, aujourd'hui, ça fait deux " (Association des Musulmans d'inspiration sunnite). D'autres sont stupéfaites par les querelles byzantines, cultivées avec autant de délices : “ Nous sommes dispersés sur l'essentiel ; si on veut régler les problèmes, c'est par là qu'il faut commencer " (AUI) ; "L'évêque d'Evreux, il y a 5 ou 6 ans de cela, il a pu obtenir un terrain pour faire construire une mosquée et avait dit aux associations de la région : "Voilà Dieu merci ! on a un terrain, on vous le donne, vous pouvez construire une mosquée, vous en avez besoin pour vos enfants... Mais on ne peut pas le donner à 15 associations, il faut donc que vous désignez ou une association, ou que vous fassiez une association particulière, ou que vous vous fédériez en une seule association. Et ça, ça n'a pas été possible et personne n'a eu le 
terrain, et il n'y a pas eu de mosquée "(VIO). L'association VIO note, d'autre part, le manque de tact ou peut-ête l'ignorance des règles du jeu de certains musulmans, qui, dans les années soixante-dix, reçurent une " grosse aide du côté catholique "; mais par la suite " ça a été perçu comme une invasion islamique au détriment du christianisme, mais souvent même des musulmans eux-mêmes n'avaient pas le tact nécessaire pour couper court à ce genre de pensée ".

L'islam, une religion pour les hommes ? Voilà, où le bât blesse pour certaines associations de femmes qui contestent cette vision, en faisant appel aux deux plus célèbres femmes du Prophète, Khadija et Aïcha, et en battant en brèche l'ignorance des mâles : « Ce sont les hommes qui nous font mal ; mais ces hommes bornés, ces analphabètes qui ne savent que répéter bêtement ce que dit un imam borné. L'islam n'a jamais privé la femme de ses droits : Khadija (première femme du Prophète) était commerçante ; Aïcha allait soigner les blessés. Ce sont les hommes qui décrètent que la femme doit rester à la maison ". Cette vision réductrice de l'islam induit à diminuer, selon certaines associations, la valeur de la religion, voire à la rendre ridicule. Elles fustigent tour à tour les pratiques ostentatoires, les réactions "problématiques" de certains musulmans lors des deux "affaires" (Rushdie, foulard). Pour ces associations, l'affaire du foulard aurait dû être réglée, au plus vite, au nom de l'islam d'ouverture ; l'affaire Rushdie, elle, a été un " scandale provoqué par les musulmans eux-mêmes. Quelqu'un qui vous attaque par l'épée, défends-toi par l'épée ; s'il attaque par un livre, réponds-lui par un livre ". Quant aux pratiques ostentatoires : " (lorsque) je vois ces gens qui se promènent avec ce « bâton ", le Siwak, arguant que le Prophète utilisait le Siwak pour nettoyer ses dents et sa gencive. C'est vrai, c'est tout ce qu'il y avait, mais si le Prophète vivait de nos jours, il utiliserait certainement le dentifrice " (Les Amis de l'Islam). Ces esquisses de discours constituent une trame sur laquelle les acteurs sociaux vont évoluer; les unes, au travers d'une " citadelle intérieure ", vont user d'attitudes ou comportements, afin de vivre et de fortifier leur foi ; d'autres vont se confronter d'une manière offensive à la société et aux institutions, en s'arrogeant le rôle de porte-parole, voire de médiateur de la " communauté musulmane "; d'autres, enfin, au nom d'un islam ouvert, ou d'une politique à long terme, vont occuper des espaces, en vue de délivrer un message.

\section{STRATÉGIES DES ASSOCIATIONS}

L'adjonction du " parler islamique " d'avec d'autres thèmes portant sur les relations entre associations, sur les salles de culte, l'éducation ou la représentation officielle de l'islam, permet de déceler les modèles d'attitudes, les stratégies, élaborées et mises en pratique par les associations. Celles-ci se structurent en quatre catégories-types, étant entendu que s'opèrent selon les contextes des glissements d'une catégorie vers une autre.

\section{LE RELIGIEUX DANS LE PRIVÉ}

Une des stratégies situe le religieux uniquement dans la sphère du privé, soit en le vivant et le pratiquant dans une "citadelle intérieure ", soit en négociant 
une ouverture avec d'autres groupes, partageant la même vision du monde. Le groupe évoluant dans une "citadelle intérieure " comprend les associations de type confrérique, structurées par les Négro-Africains, les Algériens et les Turcs. Les adeptes de ces groupes religieux - tout au moins pour les Négro-Africains, nouent avec leur maître spirituel respectif, des rapports basés sur le système de dons et de contre-dons. Chaque groupe vit à l'heure de la capitale religieuse de sa confrérie, ce sera Touba, pour les Murid, Mostaganem pour les Alawi, Konya ou Mevlana, pour les Turcs. Chez les Négro-Africains, des réunions hebdomadaires les rassemblent, réunions pendant lesquelles les adeptes participent à des séances de prière, de lecture du Coran, ou de chants religieux. Si des membres de la tijaniyya vivent en vase clos, les Murid, eux, optent pour une tactique de vulgarisation de la pensée du fondateur du mouvement, à travers une revue bilingue (français-wolof avec des caractères arabes), le Ndigel - La Voix de Murid ; de même que la Alawiya (Amis de l'Islam), qui ouvre ses séances de prières à des non-musulmans. Le deuxième exemple concerne les groupes informels - associations de fait Ahl Al Beit, et le groupe de femmes de la Mosquée de Paris. Celui-ci a opté pour une structure de type informel, parce que " chaque création d'association est une division supplémentaire; l'islam est une union vraie, qui doit être pratiquée, donc moins on crée des associations, mieux on se porte ". D'autre part, ce choix procède du souci de ne pas s'impliquer dans des activités d'ordre public, de " ne pas faire scandale ", comme " descendre dans la rue, ce qui n'est pas un comportement musulman, ni distribuer des tracts "; ce qu'il faut chercher en islam, c'est la " sérénité, le devoir accompli, c'est tout ". Le devoir accompli se vit à travers l'entraide mutuelle, tant sur le plan matériel (aider les nécessiteux, les sœurs en difficulté momentanée) que moral (visite des malades musulmans dans les hôpitaux) ; l'accès à la sérénité passe par l'étude de textes théologiques.

Le retrait du religieux dans l'ordre du privé s'applique aussi à la fois par la configuration de l'habitat (foyers de travailleurs immigrés), par l'image négative de l'islam auprès des médias, de la société civile, et par la perception d'être placé dans une relation dominants/dominés, "ils croient que nous sommes inférieurs à eux ; en France, on ne connaît pas l'islam "; et dans ce pays, plein de tentations, "tout prêt à vous dévoyer du chemin de Dieu ", les seules parades qui restent, se trouvent soit dans l'imaginaire, dans un " ailleurs spatial " (Camillieri) valorisant, "notre religion a connu des moments grandioses ", soit dans une rétroaction vers la communauté, en sacrifiant, comme l'imam-artisan d'un foyer en Seine-SaintDenis, "son temps pour être en paix avec son créateur, et d'essayer de dire aux gens que la religion est ce qui est essentiel (...) loin des baratins et de ces gens qui créent des problèmes pour eux-mêmes et pour les autres". Ce choix a sa source, d'autre part, en la nécessité de se présenter en modèle face aux jeunes qui " font des choses interdites" (vol, drogue), en leur montrant que "lorsqu'on cède aux besoins animaux, quand on quitte le chemin de Dieu, on peut tout faire ". Le troisième exemple porte sur les associations déclarées, qui sont isolées sur le plan spatial, ou au niveau de l'effectif de ses membres.

\section{DU REPLI Ã UNE POLITIQUE D'OUVERTURE}

Une autre catégorie tente de baliser des espaces, afin d'offrir une meilleure image de l'islam. En mettant l'accent " sur les revendications d'une « conscience 
islamique ", ou d'une "identité islamique ", ou en partant du "principe de ne pas rester trop sur la défensive, en s'enfermant dans le cadre immigré ", des associations essaient de "rompre les regrettables barrières" érigées par une " mauvaise compréhension réciproque entre la société française et sa deuxième communauté religieuse ". Ces associations se définissent comme site d'éducation civique, telles, l'association des serviteurs de l'Eternel, ou l'association culturelle islamique turque, qui incitent l'une à l'enseignement des droits civiques aux enfants et jeunes, et l'autre, à la "nécessité d'apprendre la culture de la France ", tant aux jeunes qu'aux adultes; la pédagogie de la comparaison, a, semble-t-il eu des effets bénéfiques sur ces jeunes Turcs " agressifs ", devenus " calmes " et sensibles aux thèmes tournant à " si ces gens (les Français) avaient fait dans notre pays ce que vous faites ici, vous ne serez pas contents, comme eux ".

\section{STRATÉgIE OFFENSIVE ET APPEL Ã LTSLAM}

La stratégie des associations peut être parfois offensive. Elle s'arroge le rôle d'intermédiaire de la communauté islamique et use de la "fonction tribunicienne " (G. Lavau, 1981 ; R. Leveau, 1989) auprès des pouvoirs publics, et de la société civile. Elle se veut gardienne jalouse de l'image et de l'alimentation des populations musulmanes, sujettes aux divisions nationales, ethniques, et aux idéologies internationales. Certaines associations se donnent la mission de structurer une communauté, par le biais d'un enseignement religieux auprès de ses membres, et d'un mode de vie basé sur celui du Prophète (Foi et Pratique). Principale composante de l'islam en France, la Jamaat se considère comme un lieu de décantation, où les gens viennent, se purifier, et peuvent opter entre rester ou partir constituer une autre structure. Actuellement, elle rassemble essentiellement des Maghrébins - en majorité marocaine, même si l'Émir est d'origine tunisienne, des Négro-Africains, plus particulièrement de l'ethnie soninke, des Pakistanais... Optant pour une socialisation à la base, la Jamaat investit tous les lieux de vie : cafés, rues chaudes, hôpitaux, foyers de travailleurs immigrés, ilôts sensibles, métro... Toute rencontre est perçue comme une mission.

\section{LA PART FÉMININE}

En général, "l'observance féminine de l'islam " s'avère difficile. Mises à l'écart selon le principe de la division du travail social, les femmes se retrouvent reléguées de fait du domaine religieux public, contrairement aux " textes sacrés islamiques" (Andézian, 1990).

En France, des Algériennes, adeptes d'un ordre soufi, la Issawiyya (fondée au $\mathrm{XVI}^{\mathrm{e}}$ siècle au Maroc), mènent toutefois une vie religieuse intense dans leurs pratiques quotidiennes, mais aussi bien à l'occasion des rites de passage (naissance, circoncision, fiançailles, mariage, décès) que lors de rencontres hebdomadaires de prières, et pendant " les fêtes canoniques et le ramadan ". Du côté des Turcs, les femmes sont représentées dans des sections spécifiques au sein d'associations islamiques, comme l'Union Islamique de France, par exemple ; chez les Négro-Africains, des Sénégalaises, membres de la confrérie murid participent, 
selon les régions, soit à des cercles religieux mixtes, soit à des regroupements autonomes.

L'islam en France se conjugue aussi au féminin ; sur un échantillon de 74 associations, 5 d'entre elles sont exclusivement féminines. Implantées dans les Hauts-de-Seine ( 3 associations) et à Paris (2), ces associations ont été créées entre 1978 et 1986, comme l'indique le tableau ci-dessous :

\begin{tabular}{c|l} 
Date de création & \multicolumn{1}{|c}{ Associations } \\
\hline 1978 & Union française des Femmes musulmanes \\
1982 & Groupe informel des Femmes de la Mosquée de Paris \\
1984 & Union des Femmes arabes en France \\
1985 & Femmes, familles musulmanes \\
1986 & Solidarité musulmane
\end{tabular}

Au niveau des nationalités, deux associations sont franco-françaises, l'Union française des Femmes musulmanes, le Groupe informel des Femmes de la Mosquée de Paris, et la troisième est franco-libanaise (Union des femmes arabes en France). Quant aux deux autres associations, l'une est britannique, et la seconde est marocaine. Toutefois, dans les faits, toutes ces associations déclarées (4) ou informelle (1), regroupent d'autres nationalités, algérienne, marocaine, sénégalaise, syrienne, irakienne...

\section{DISCOURS SUR LISLAM}

3 associations veulent "remontrer le vrai visage de l'islam " (UFAF) qui n'est ni la " violence ", ni le " tchador "; le groupe informel stigmatise l'attitude des musulmans qui participent aux manifestations " descendre dans la rue, ce n'est pas un comportement de musulman, ni distribuer des tracts; il faut laisser les places publiques à leur usage ; on ne doit pas faire scandale ; il y a juste quelques ténors qui font beaucoup de bruit ". L'Union française des femmes musulmanes invite à la vigilance " il ne faut pas que la communauté se fasse manipuler par les intégristes ; il faut rendre les musulmans de France vigilants face aux influences extérieures. Nous voulons un islam français, pas importé ".

Si " le musulman a le devoir de respecter les lois du pays", les autorités à leur tour, doivent reconnaître la culture et la religion des musulmans, en " libérant le problème du voile pour les photos d'identité ", en octroyant le "droit à une aumônerie officielle ", en développant l'enseignement de l'arabe " au même titre que l'espagnol et le portugais ", et en réglant la question de l'abattage rituel " c'est un problème car ça fait partie du rite; en fait on ne sait où égorger; les autorités se rejettent chacune les responsabilités" (Groupe informel). 
Certaines associations (3) estiment que "l'islam a libéré l'homme de l'homme" (Solidarité Musulmane), que "dans le Coran, la femme est née libre "(FFM), et que la " femme a beaucoup de droits, de signer, d'acheter sans la permission de son mari " (UFAF). Si la société des mâles a perverti l'essence de la religion, la femme, de son côté, y a contribué " quand je vois ces femmes ignorantes, avec 8 gosses et le mari en chômage, ça me choque. L'islam n'a jamais dicté à la femme maghrébine de pétrir le pain et de fabriquer des gosses, ni de servir l'homme. La responsabilité des enfants incombe à l'homme et à la femme ; il faut vraiment que les femmes prennent conscience de leur responsabilité " (FFM).

\section{DU LOCAL AU NATIONAL}

Insérées ou non dans le tissu local ou national, les associations à caractères religieux, interrogent, de par leur particularisme, les institutions, qui étaient habituées jusqu'alors à traiter de problèmes ou demandes connus et circonscrits dans leur champ d'action.

En bouleversant l'agencement du réseau associatif de type classique, elles remettent en question le jeu relationnel préétabli, et les domaines attribués, à savoir le social, l'entraide et le culturel ; et selon les lieux, les sensibilités, ces associations vont être bien ou mal acceptées; leur présence va être bien ou mal interprêtée, ou prise en considération comme facteur de structuration et de socialisation.

\section{COHABITATION ET RAPPORT AVEC LES AUTORITÉS LOCALES}

Dans leur mise en relation avec l'environnement immédiat, les associations situent les problèmes rencontrés à deux niveaux. Celles qui disposent d'un lieu de culte se sont confrontées à des remarques du genre envahissement ou invasion ; il est à noter que seules les associations draînant beaucoup de monde, les vendredis et jours de fêtes sont sujettes à ces considérations. Toutefois, cette gêne n'a pas encore généré de situation conflictuelle entre habitants du quartier, ou usagers et les responsables associatifs, sauf celui de l'association Jamaat Fath, qui, tout au début des activités de son organisme, a été convoquée au commissariat du quartier, sur plainte des voisins ; mais depuis lors, si la cohabitation n'est pas conviviale, elle n'est pas non plus tendue, puisque certains habitants du quartier admettent que leur " territoire " n'est plus fréquenté par des populations "marginales" (prostituées, vendeurs de drogue, délinquants).

Si l'abattage rituel entraîne depuis l'émission de Brigitte Bardot (janvier 1990 sur TF1) une levée de boucliers, les associations ne semblent pas avoir été l'objet de menaces ou de remarques désobligeantes. La grande majorité d'entre elles note la difficulté de procéder à un sacrifice rituel lors de l'Ayd; elles reconnaissent avoir trouvé des méthodes discrètes pour ne pas ameuter le voisinage : certaines associations accomplissent le rituel dans des fermes ; d'autres, 
dans des caves aménagées officiellement dans les foyers de travailleurs ; et d'autres, enfin, se rendent aux abattoirs, ouverts pour la circonstance. En dépit des aléas, les associations - à quelques exceptions - semblent épouser les contours de la société d'accueil, et optent pour une politique de la discrétion.

La politique de décentralisation des années 80 a induit un " mouvement de territorialisation de l'action publique ". Les municipalités voient leur pouvoir accru, dans une situation économique difficile ; ceci va jouer sur les rapports entre autorités locales et mouvement associatif dans son ensemble, en accentuant dans nombre de cas le clivage entre nationaux et étrangers. C'est dans cette période incertaine qu'a commencé à éclore et surtout à se développer le mouvement associatif islamique. Dans l'ensemble, les associations de l'échantillon entretiennent des relations suivies, plutôt bonnes (déclarent 45 associations) que mauvaises (pour 15 associations) ou quasi inexistantes (14 associations). Les différents types de relations ne suivent aucun clivage d'ordre politique ; certaines municipalités de sensibilité de gauche ou de droite peuvent être, soit réceptives au dialogue, soit refuser tout échange. Les associations qui entretiennent de bonnes relations avec les institutions locales mettent en avant l'aide apportée pour la recherche d'un local ou d'un terrain. C'est la majorité. L'autre partie est plus sensible aux rapports sociaux ("c'est une chance d'avoir un maire qui connaît les Maghrébins ") et à l'esprit d'ouverture et de concertation du maire, comme le note l'association culturelle islamique " le but de la création de cette association musulmane est la construction d'une mosquée, le maire m'a demandé de créer une association avec un projet valable ". Parmi celles qui ont des rapports quasi inexistants se trouvent des associations déçues par une municipalité qui a tenté de contrarier toute activité de type religieux. D'autres, en revanche, n'ont même pas tenté de nouer le contact, soit parce qu'elles "vivent en vase clos ", et n'éprouvent pas jusqu'ici la nécessité d'entrer dans un jeu relationnel, soit parce qu'une association de même type a été reconnue comme le porte-parole de la " communauté ", ou alors, étant des associations de fait, elles ont préféré explorer d'autres champs moins institutionnels. Les associations n'ayant pas de bons rapports avec leurs municipalités spécifient que celles-ci, soit refusent de les recevoir ou de leur allouer un local, soit ne tiennent pas leurs promesses, ou alors usent du droit de préemption ou font les règlements d'une manière drastique appliquer.

\section{LA QUÊTE DE LA REPRÉSENTATIVITE ET PERCEPTION DU CORIF (CONSEIL DE REFLEXIONS SUR L'SLAM EN FRANCE)}

" 11 faut institutionnaliser l'islam en France pour éviter les récupérations ", déclare l'Association des étudiants islamiques de France. L'idée d'une représentation officielle semble recueillir les suffrages des associations : " c'est un vœu légitime ", pour les unes, une " nécessité » pour les autres. Si la quasi unanimité s'opère autour d'une représentation de l'islam, en revanche lorsque la question des critères de choix est posée, là apparaissent des clivages, voire des divergences. Quatre cas de figure sont en présence.

Une petite minorité privilégie les États d'origine, qui, à leur tour, nomment 
des intermédiaires. Pour une association - très représentative -, "c'est à la communauté de choisir la forme selon laquelle il faut s'organiser ".

Pour une quinzaine d'associations, seules "les associations musulmanes décidées à s'intégrer " peuvent être choisies, parce qu'elles "connaissent les maux dont souffrent les associations". La grande majorité des associations mettent l'accent sur les critères religieux, d'abord : des personnalités de " foi ", de "piété ", ayant une " solide connaissance de l'islam ", totalement " compétentes ", " intègres ", "désintéressées ", d’une grande " ouverture " et " tolérance ", " motivées " et " défenseurs des intérêts de la communauté ". Ensuite, elles doivent avoir l'étoffe de "véritables dirigeants ", c'est-à-dire d'être " des bergers qui ne tuent pas leur bétail ".

Sur un échantillon de 55 associations - il y a eu 13 non-réponses -42 d'entre elles sont au courant de la création du Corif, soit parce qu'elles ont été convoquées pour information à la Préfecture (6 d'entre elles), soit parce qu'elles ont reçu le renseignement d'un ami, d'un " frère " (30), soit parce qu'elles en sont membres (6). Il est à noter que les associations de fait ( 2 en Seine-Saint-Denis) ont été convoquées, elles aussi, à la Préfecture ; mais selon le dirigeant d'une de ces associations, il y a eu méprise, car son association était considérée comme déclarée.

Le Corif est jugé favorablement, même par ceux qui n'étaient pas au courant de sa création ; reste cependant une certaine ambiguïté : la majorité des associations sont convaincues que le Corif est un organisme officiel, et que par sa création, l'État français reconnaît enfin l’islam.

Si ce sentiment perdure, il risquerait à moyen terme de générer un effet pervers. Tant d'attente déçue ferait doublement le jeu de certains États, dépossédés momentanément de leur légitimité, et d'associations, très critiques dès le départ à l'égard de cet organisme, " ce n'est pas au gouvernement de désigner tel ou tel représentant ", et à l'égard de ses membres, perçus comme des "fantoches ", " non représentatifs", des "cadres imposés".

Ou alors ce sentiment est-il entretenu par les membres du Corif eux-mêmes, entraînés qu'ils sont dans le "vertige institutionnel "? La base est, quant à elle, pleine d'espoir, et se dit " si les gens sont de vrais musulmans, qu'ils se mettent d'accord sur l'essentiel, et qu'ils viennent nous voir, nous informer pour que nous puissions à notre tour informer les musulmans des mosquées qui ne connaissent rien, mais qui sont concernés (AUI).

\section{ASSOCIATIONS ET INTÉGRATION}

Le terme "intégration " a été diversement apprécié et commenté par une dizaine d'associations. Si des associations et fédérations ne souscrivent pas entièrement à l'idée selon laquelle, "l'intégration, c'est l'éloignement de l'islam ", elles estiment, toutefois, que ce concept d'intégration prégnant d'ambiguïté et de confusion, est souvent appréhendé dans le sens d'un " gommage des spécificités 
culturelles", d'une "désintégration de notre culture pour s'intégrer dans une autre culture ". Et partant, elles s'insurgent contre pareille approche "On est pour l'intégration, mais pas celle qui signifie pour beaucoup assimilation " (UOIF) ou "se faire presque chrétiens pour faire plaisir aux Français" Intégrité).

Pour les Amis de l'islam, l'ambiguïté est patente " quand on nous demande de reléguer la religion dans la sphère du privé ", et cette association de se demander " est-ce que cela signifie qu'il s'agit de la pratique cultuelle de la religion ou est-ce qu'ils parlent de ce qu'on vit à travers la religion ? Ces associations proposent en lieu et place de " participer à la vie française, tout en gardant les spécificités, à la fois culturelles et cultuelles ", dès lors qu'il " n'y a nullement incompatibilité entre le fait d'être musulman et de vivre dans un milieu qui ne l'est pas " (Amis de l'islam). Mais pour ce faire, les musulmans, eux-mêmes, doivent se définir, en optant pour une solution " soit qu'ils restent ici, et (alors), ils doivent s'intégrer, ne pas être en contradiction avec les lois sociales ; soit, choisir de repartir et vivre dans leur pays "(UOIF). S'ils décident de vivre en France, leur " adaptation aux normes sociales ne doit (cependant) pas se faire aux dépens de leur identité culturelle " (UOIF), et la " cohabitation avec les Français " ne devra pas signifier, pour une association turque, une sorte de transaction du genre "la première génération est à vous, mais la seconde, nous la gardons pour nous ". Pour Mevlana, une autre association turque, l'intégration bien comprise de part et d'autre "peut être réalisée, si les hommes se respectent entre eux "; du reste, l'expérience a déjà opéré de manière satisfaisante en Turquie, car " dans notre pays, les croyants de différents pays vivent depuis des années en parfaite intégration "; aussi un tel modèle pourrait-il servir ailleurs, " nous, ici, nous allons faire un effort allant dans ce sens, dans la mesure où on nous respecte dans le cadre des lois ". Pareil respect, d'après une association comorienne, " ça commence dans le quartier, devant la porte de l'école, devant la porte de la mairie, devant la queue de la préfecture ", mais les musulmans, aussi, " ne doivent pas réclamer l'islam comme s'ils étaient dans un pays islamique ", précise l'UOIF, qui invite à plus de pragmatisme et d'accomodement " si l'islam se présente comme un mode de vie complet, c'est vrai dans une société musulmane, mais dans une société non musulmane, la religion doit s'appliquer au niveau individuel et communautaire ; on ne peut pas demander à la société française d'observer, d'adopter, les obligations de l'islam ".

Pour la majorité des associations (65), la participation associative "favorise ", " renforce l'intégration " parce que " c'est nous qui connaissons nos maux, et qui pouvons aider à les résoudre ".

L'association, comme lieu de rencontre, permet la structuration des membres, d'aplanir les différences, et de rompre la barrière générationnelle ; en tant que moyen pour une ouverture sur l'autre, elle donne un " bagage qui permet de devenir un élément positif dans la société "; ce " bagage " comprend une éducation religieuse destinée aux adhérents, parce que, très souvent, "ils ignorent l'islam et ses valeurs "; par un travail d'explication, "nous luttons contre toutes les malfaisances que notre religion elle-même interdit "; "l'adaptation " à la société implique d'une part la " nécessité d'apprendre la culture de France", 
d'" utiliser la langue française comme langue principale ", d'autre part, afin de "maintenir les rapports entre frères et les non-musulmans". Ce lieu d'apprentissage des responsabilités favorise une " certaine cohésion des gens, et va ensuite " influer directement sur les liens sociaux, en général "; le " sentiment social ", pour être viable estiment une douzaine d'associations doit reposer sur la loi islamique, puisque « le but de l'islam sur le plan social, c'est d'unir les hommes entre eux ", et, si la leçon est retenue, " cela conduit normalement à aider à l'intégration ".

Le mouvement associatif aide en outre à la socialisation des enfants : " l'autre jour un père d'un enfant m'a appelé dans la rue pour me dire que son fils (8 ans) qui vient à l'association (pour des cours d'arabe et de religion), est plus sage et travaille bien à l'école et mieux que ses autres frères ". Ce sentiment d'apporter une " contribution éducative " complémentaire et non antinomique à l'enseignement laïc est très largement partagé par les associations ; certaines sont même convaincues que l'absence de repères dont souffrent une catégorie de "beurs, complètement déphasés, complètement désorientés " s'explique par leur distanciation par rapport à l'éducation religieuse ; et pourtant, "si on nous donne les moyens, nos droits, nous, on fait nos devoirs, et alors c'est " la France qui va en profiter, si on aide les gosses à réussir, et devenir demain des ingénieurs".

D'autres associations -- au nombre de 4 - tout en reconnaissant la dimension intégrative du phénomène associatif, mettent en exergue ses limites. "C'est un moyen d'intégration, mais ce n'est pas suffisant, parce que les immigrés sont rejetés par la société française ; parce qu'ils n'ont pas le droit de voter, le droit de revendiquer leurs besoins ". Raison de plus, semblent répondre une quinzaine d'associations, pour " fréquenter l'association, apprendre ici ce qu'on ne savait pas auparavant "; pour " mieux définir notre point de vue en se comparant les uns aux autres ", pour être " conscientisés ", et " essayer d'améliorer la mentalité qui règne chez les musulmans ".

Nationaux de souche, naturalisés ou en voie de l'être, étrangers provisoires ou définitifs, tous semblent d'accord sur l'aspect heuristique du fait associatif. Repli communautaire sur la base du religieux ? Une première lecture pourrait le laisser transparaître, et les activités d'un mouvement piétiste, telle la Jamaat Tabligh (Foi et Pratique) renforcerait cette vision des choses. La participation associative est un élément important dans le processus vers l'intégration des étrangers ; en tant que lieu d'éducation, même ou surtout religieuse, l'association génère un " sentiment social ", et permet l'apprentissage des responsabilités, et la découverte de l'autre. Pour rencontrer une culture exogène, il est nécessaire d'appréhender d'abord la sienne.

\section{CONCLUSION}

Le réseau associatif immigré a connu, en général, diverses étapes dans son existence ; d'informel - le lien parental, villageois, voire régional, servant souvent d'aune --, il s'est, par la suite, structuré en organisations institutionnalisées, inter- 
médiaires entre les acteurs sociaux en procès de stabilisation et les autorités locales et nationales.

Se définissant comme sites de mobilisation des ressources identitaires, les associations de type laïque, dans leur transaction avec les instances de la société d'accueil, ont élaboré et mis en chantier des schémas d'expression culturelle et politique ; et ses dirigeants ont excipé de médiateurs socio-culturels, et de décodeurs des attitudes et comportements de leurs compatriotes.

Par sa présence dans le champ associatif, une autre instance de médiation, aux contours à la fois culturels et cultuels, introduit une sédition dans cette ordonnance, en s'arrogeant le double rôle de gestionnaire de l'éducation des jeunes issus de l'immigration, de leurs parents, et de représentante et défenderesse des intérêts de l'islam en France. Pour ces associations de type religieux, l'éducation est appréhendée comme un "phénomène social total "; elles développent des activités socio-culturelles, tentent de diffuser et de valoriser la culture islamique, engagent différents types de négociations avec les pouvoirs publics, et esquissent des politiques pour entrer en concurrence avec, voire battre en brêche le modèle des associations de type laïque. En construisant des nouvelles frontières de l'identité, les organisations religieuses induisent-elles la marginalisation des populations musulmanes ou favorisent-elles une forme d'intégration sociale ? Telle était notre problématique ; qu'en est-il, au terme de cette étude?

Mais d'abord quelques constats révélés par l'enquête. Au niveau des motifs de création d'associations, la loi d'octobre 1981 portant sur la libéralisation des associations étrangères a joué un rôle déterminant dans ces créations, même si elle n'a pas été le seul facteur déclencheur ; cette loi, d'autre part, semble avoir privilégié celle du $1^{\text {er }}$ juillet 1901 , relative au contrat d'association aux dépens de la loi du 9 décembre 1905, concernant les associations religieuses. La méconnaissance de la loi 1905 pourrait amplifier la confusion entre la sphère du public et celle du privé ; une meilleure compréhension des deux lois - 1901 et 1905 - pourrait inciter les acteurs sociaux musulmans à créer deux structures bien distinctes, l'une cultuelle, et l'autre socio-culturelle, à même de bénéficier d'éventuelles subventions octroyées par les pouvoirs publics (FAS, par exemple).

S'agissant de la perception de l'islam, on se trouve en face de deux attitudes, grosso modo : d'une part des musulmans critiquent d'autres musulmans qui, en ignorant leur religion, en cultivant le "narcissisme des petites différences ", en donnant dans des pratiques ostentatoires et en manipulant le religieux par le politique, dévoient l'esprit de l'islam et sont donc en grande partie responsables de l'image négative véhiculée sur leur religion ; d'autre part, des musulmans — une majorité - s'insurgent contre le portrait d'un islam violent et intolérant dressé par la société française, et s'interrogent sur un État de droit, qui tente d'« essentialiser " l'islam, selon l'expression de J.L. Schlegel, " c'est-à-dire d'en faire une religion définitivement et intrinsèquement différente, définitivement fermée à la modernité politique, sociale, culturelle ". Toutefois, au niveau local, même si des problèmes de relations et de cohabitation existent - et ils existent (local, lieu de culte, ayds) - le propos défensif et accusateur des associations est plus nuancé dans la plupart des cas. 
Au niveau des appartenances, les associations donnent la prééminence à la nationalité, voire à l'ethnie : le sentiment collectif (appartenance à la umma) semble plutôt lâche, malgré les tentatives de regroupements associatifs, et malgré les alliances objectives nouées selon les conjonctures. Au-delà des clivages nationaux apparaissent des différenciations d'ordre confrérique, ou idéologique ; la course à la représentativité amplifie les clivages, et la mise en place du Corif a jeté une lumière crue sur ces dissensions.

Les associations au travers des modèles d'attitudes, se structurent en quatre catégories - étant entendu que pourraient s'opérer selon les contextes des glissements d'une catégorie vers une autre. La première d'entre elles situe le religieux dans la sphère du privé, soit en le vivant et le pratiquant uniquement dans une " citadelle intérieure ", soit en négociant en même temps une ouverture avec d'autres groupes partageant la même vision du monde, ou en optant pour une tactique d'explication et de vulgarisation de la pensée du fondateur du mouvement. Confréries, et certaines associations isolées - sur le plan spatial - se situent dans cette mouvance. La seconde catégorie tente de baliser des espaces, afin de présenter une meilleure image de l'islam; elle met l'accent sur la diffusion d'un enseignement portant sur des valeurs universelles (fraternité, liberté, tolérance, justice, paix, famille), s'implique dans un dialogue islamo-chrétien, et pratique la politique " portes ouvertes ", à l'occasion des fêtes musulmanes (Ayds) ; quant à la troisième, en revendiquant une identité islamique, elle essaie de jouer le rôle d'intermédiaire de la " communauté ", et use de la " fonction tribunicienne " auprès de la société civile, et des pouvoirs publics; gardienne jalouse de l'image et de la consommation alimentaire des populations musulmanes, elle place son action sous le signe d'une politique offensive afin de faire émerger une " conscience islamique ". La dernière catégorie, en se donnant pour mission de structurer ses membres par le biais d'un enseignement et de pratiques suivant le modèle du Prophète, propose une manière autre de vivre l'islam, en faisant fi des contingences de la vie quotidienne - en principe, en tout cas.

L'imam occupe dans la structure associative une place prépondérante, d'autant plus que niveau d'éducation religieuse des dirigeants est en général relativement faible. Outre ses fonctions de guide des prières, il assure aussi le rôle d'officier d'État civil pour les mariages religieux, d'enseignement de langue, de théologie et de culture islamique, celui d'intermédiaire et confident auprès des familles, et de maître spirituel pour les adeptes de certaines confréries, négro-africaines, en l'occurence. L'islam, par ailleurs, se décline au féminin de manière souterraine (cas de l'ordre religieux Issawiya) et de manière plus affichée, par le biais d'associations déclarées ou de fait. Les dirigeants des associations enquêtées sont à la fois bien insérés dans le tissu socioprofessionnel, et tout aussi décidés à faire admettre la part féminine dans le mouvement associatif de type religieux, parce que conscientes d'être le vecteur principal de la transmission des valeurs socio-culturelles, parce que prêtes "à s'intégrer tout en restant soi-même ".

Dans Tristes Tropiques, C. Levi-Strauss définissait l'islam comme " grande religion qui se fonde moins sur l'évidence d'une religion que sur l'impuissance à nouer des liens au dehors ". Proposant un début de réponse à cette formule lapidaire, des associations situent leurs actions dans le cadre d'une société civile 
laïque ; certaines, partant de l'adage " mens sana in corpore sano " investissent dans le sport collectif (football) et individuel (équitation, arts martiaux), dans le double objectif de structurer, de contrôler les jeunes, et de tisser des liens avec l'environnement ; d'autres, pour éviter toute forme de marginalisation, organisent des sorties guidées, afin de s'imprégner des valeurs socio-culturelles de la société d'accueil ; il est des associations qui optent d'attendre la fin des études primaires pour initier les enfants aux langues d'origine, afin d'éviter que cette " éducation soit faite au détriment des études à l'école française en créant un élément d'hésitation chez les enfants "; d'autres, encore, dispensant des cours de soutien et d'éducation religieuse - deux objectifs perçus comme complémentaires, et non antinomiques ; il est, enfin, des associations qui, dans le cadre d'une meilleure intégration de ses membres, débattent en français - devenu langue de travail et de communication -, des problèmes de société, au cours de journées de rencontres ou de colloques annuels. Autant d'indices dévoilent la " ruse d'intégration " mise en essai par la majorité des associations. Certes, nombre d'entre elles restent tributaires des politiques de leurs États d'origine, et une minorité, des subsides provenant jusqu'à récemment de certains États étrangers (pays du Golfe); cette situation de précarité et d'instabilité pourrait constituer un frein au désir d'intégration, de même que l'externalisation de l'islam mise en pratique par les différents gouvernements français, du reste en contradiction avec les visées d'une constitution d'un "islam gallican ". Si la tendance dominante vers un processus d'intégration est réelle parmi les associations étrangères - il faut garder à l'esprit que près de la moitié des dirigeants est française, de souche ou d'adoption -, il reste toutefois une petite minorité, étrangère et autochtone, pratiquant une lecture littérale des textes, pour lequel, tout accomodement à la société laïque constitue, soit un danger, soit un deni de justice.

Le devenir des associations islamiques va être sujet à questionnement ; dici quelques années va se poser le problème de la relève, tandis que pour les associations de type laïque, l'on assiste à l'émergence de jeunes dirigeants. Va-t-il s'opérer une interaction entre les deux structures ? Le laïque va-t-il investir le religieux pour le transformer, ou être transformé par lui ? L'autre cas de figure, c'est que des jeunes formés religieusement reprennent le flambeau, et selon l'éducation reçue, reproduisent les mêmes catégories de penser et les mêmes stratégies que leurs anciens maîtres; ou bien s'appuyant sur leur double formation, laïque et religieuse, ils établissent soit des passerelles entre le profane et le sacré, soit, ils investissent les associations de type laïque, pour les transformer en appendices d'associations religieuses, mais avec l'hypothèse sous-jacente d'une éventuelle récupération du religieux par le laïque. 


\section{Notes et références bibliographiques}

* Synthèse du Rapport réalisé par le Centre d'Études et de Recherches Internationales. Fondation Nationale des Sciences Politiques dans le cadre de la convention d'étude avec le Ministère de la Solidarité, de la Santé et de la Protection sociale. Direction de la Population et des Migrations.

\section{LISTE DES ABREVIATIONS}

F.N.M.F. : Fédération Nationale des Musulmans de France.

F.A.I.A.C.A. : Fédération des Associations Islamiques d'Afrique, de Comores et des Antilles.

U.O.I.F. : Union des Organisations Islamiques en France.

A.E.I.F. : Association des Etudiants Islamiques en France.

A.C.I.E. : Association Culturelle Islamique d'Ecquevilles.

A.M.I.S. : Association de Musulmans d'Inspiration Sunnite.

F.F.M. : Femmes, Familles Musulmanes.

M.I.M.E. : Mouvement Islamique des Mourides en Europe.

U.I.F. : Union Islamique de France.

V.I.O. : Vivre l'Islam en Occident.

A.I.C.M.S.F. : Association Islamique et Culturelle des Marabouts Sénégalais en France.

A.C.M.C.S. : Association Culturelle des Marabouts - Casamance (Sénégal).

A.I.C. : Association Islamique Comorienne.

A.R.A.I. : Association pour la Rénovation de l'Alliance Islamique.

U.I.C. : Union Islamique Comorienne.

A.I.F.J. : Association Indo-Française des Jaffari.

A.C.I. : Association Culturelle Islamique.

A.C.M.I.F. : Association Culturelle des Musulmans d'Ile-de-France.

A.C. IBN AFFANE : Association Culturelle Othman IBN AFFANE.

A.U.I. : Association d'Unification Islamique.

\section{REVUES ET JOURNAUX}

- L'Index, publication de l'association Intégrité, 1989-1990.

- Nouvel Observateur, 19-25 juillet 1990.

- Revue du Monde Musulman, janvier-mai 1908.

\section{ARTICLES ET OUVRAGES}

ANDEZIAN (S.), 1990. Lislam au féminin, Migrations-Société, vol. 2, no 9-10, mai-août, p. 7-16. BRISEBARRE (A.M.), 1989. La célébration de l'Ayd El Kébir en France : les enjeux du sacrifice, Archives des Sciences Sociales des Religions, 68/1, p. 9-25.

DASSETTO (F.), 1988. Le Tabligh en Belgique. Diffuser l'Islam sur les traces du Prophète, Louvain, Sybidy papers. 
DIOP (A.M.), 1985. Les associations murid en France, Esprit, juin, p. 197-206.

1989. Immigration et religion : les musulmans négro-africains en France, Migration, Société, vol. 1, no 5-6, octobre-décembre, p. 45.57.

ETIENNE (B.), 1987. L'Islamisme radical, Paris, Hachette.

HAMES (C.), 1979. Islam et structures sociales chez les immigrés soninke en France, Social Compass (26), 1, p. 87-98.

K RAMER (M.), 1986. Islam Assembled. The Advent for the Muslim Congresses, N.Y. Columbia University Press.

KASTORYANO (R.), 1988. Paris-Berlin : Politiques d'immigration et modalités d'intégration des Turcs in R. Leveau et G. Kepel (ed) Les Musulmans dans la Société française, Presses de la Fondation Nationale des Sciences Politiques.

KEPEL (G.), 1987. Les banlieues de I'Islam. Naissance d'une religion en France, Paris, Ed. Seuil. 1990. La Revanche de Dieu, chrétiens, juifs et musulmans à la reconquête du monde, Paris, Seuil.

LAVAU (G.), 1981. A quoi sert le parti communiste français? Paris, Fayard.

LEVEAU (R.). Les partis et l'intégration des "Beurs ", in Mény (dir.) - Idéologies, partis politiques et groupes sociaux (études réunies pour Georges Lavau).

LEVEAU (R.) et KEPEL (G.) (dir.), 1981. Les musulmans de la société française, Paris, Les Presses de la Fondation Nationale des Sciences Politiques.

WITHOL DE WENDEN (C.), DIOP (A.M.), BAROU et alia, 1986. Analyse des conflits récents survenus aux usines Renault de Billancourt depuis 1981 au sein de la population immigrée. Contrat de connaissance CNRS/RNUR. 


\section{Le mouvement associatif islamique en Ile-de-France}

\section{A. Moustapha Diop et Riva Kastoryano}

L'émergence de regroupements investissant dans le champ du sacré constitue une nouvelle donne dans l'espace associatif immigré. Ceux-ci se définissent comme sites des ressources identitaires, et, dans leur transaction avec les instances de la société d'accueil, élaborent et mettent en chantier de nouveaux schèmes d'expression culturelle, politique, symbolique. Les dirigeants de ces associations, en s'arrogeant le rôle d'" entrepreneurs identitaires ", amorcent-ils une politique de marginalisation voire de repli des populations musulmanes, ou favorisent-ils un modèle d'intégration sociale?

L'article, après avoir retracé la genèse et les raisons de création de ces associations, analyse la structuration des appartenances, sinterroge sur les stratégies amorcées en vue de la transmission de la culture, langue et religion, et, d'autre part, rend compte des différents types de discours et des rapports entretenus au niveau local et national.

\section{Associative islamic movement in Ile-de-France}

\section{A. Moustapha Diop et Riva Kastoryano}

The growth of religious organisations has become a new issue as far as migrants groupings are concerned in France. The former défined themselves, as the bulk identity makers of the muslim migrant populations, and thus, try and set the line, by using various types of policy, either in the cultural field or in the political, say, symbolic ones, when negociating with the authorities at every rung of the ladder. By acting in such a way, are they building barriers between the muslim migrant populations and the native population, or rather, are they are trying to find ways and means in order to make the muslims tope with the guest society?

The paper deals with different aspects of muslim organisations : history and reasons of creation of the organisations ; structuring of belongings ; ways of communicating culture, language, religion ; discourse on islam and its perception : relationship at local and national level.

\section{El movimiento asociativo islámico en Ile-de-France}

\section{A. Moustapha Diop et Riva Kastoryano}

La aparición de agrupaciones que invierten en el campo de lo sagrado constituye una nueva situación en el àmbito asociativo de la inmigración. Estas agrupaciones se definen como un espacio de recursos de identidad y en su trato con las instancias de la sociedad que las acoge, elaboran y ponen en obra nuevos esquemas de expresión cultural, politica y simbolica. Los dirigentes de estas asociaciones, al atribuirse el papel de "contratistas de identidad", ¿ acaso no inician una política de marginalización e incluso de repliegue de la población musulmana o, por el contrario, favorecen un modelo de integración social?

El artículo, tras describir el génesis y las razones de creación de estas asociaciones, analiza la estructuratión de las adhesiones, se plantea cuestiones sobre las estrategias iniciadas con vistas a la transmisión de la cultura, lengua y religión, y por otra parte, da cuenta de los distintos tipos de discursos y de las relaciones mantenidas en el ámbito local y nacional. 